\section{Case Reports in Orthopedic Research}

Case Rep Orthop Res 2020;3:108-117

\title{
Fracture Dislocation of the Anatomical Neck of the Proximal Humerus: Case Report and Literature Review
}

\author{
Joseph Maalouly Dany Khalil Aouad Antonios Tawk Georges El Rassi \\ Department of Orthopedic Surgery and Traumatology, Saint Georges University Medical \\ Center, University of Balamand, Beirut, Lebanon
}

\section{Keywords}

Proximal humerus · Fracture - Dislocation · Trauma

\begin{abstract}
Fractures of the anatomical neck of the humerus are an extremely rare pathological entity in the field of orthopedic surgery. One of the most feared complications associated with anatomical neck fracture of the humerus is avascular necrosis (AVN). The literature does not contain clear, evidence-based guidelines for the surgical management. This case report highlights the case of a 33-year-old male patient who presented with anatomical neck humerus fracture dislocation after a snowboarding accident. Computed tomography showed a comminuted displaced fracture involving the right humeral neck associated with anterior dislocation of the right shoulder. A small bony fragment adjacent to the glenoid posteriorly was also revealed. The patient was treated with open reduction and internal fixation (ORIF). After 3 years of follow-up, the patient is fully recovered with a normal range of motion of his right shoulder joint. The patient did not show any signs of AVN of the humeral head throughout the course of the 3-year follow-up. Anatomical neck fractures of the humerus are uncommon injuries in the literature. Due to the poor vascularization and the absence of attachment to soft tissue, there is a significantly high risk of AVN associated with fractures of the anatomical neck of the humerus. The patient presented in our report was treated with ORIF since it allows preservation of the humeral head.




\section{Case Reports in Orthopedic Research}

\section{Introduction}

As per the literature, fractures at the level of the anatomical neck of the humerus are uncommon injuries encountered in the field of orthopedic traumatology, accounting for around $0.5 \%$ of all proximal humerus fractures [1]. On the other hand, anterior shoulder dislocations secondary to trauma are common injuries, with an incidence of around 40 per 100,000 persons in the USA [2]. Among the young active population, the recurrence risk approaches $90 \%$ [3]. Henceforth, early operative management of the dislocation is oriented towards the restoration of glenohumeral stability and decreasing the risk of future recurrence of dislocations to $6-23 \%[3,4]$. Therefore, anterior shoulder dislocation associated with anatomical neck fracture of the humerus secondary to trauma is not only a rare, but also a complicated injury. The literature describes various surgical methods for the management of such injuries; however, the nonsurgical management of such injuries is not recommended [5-7]. Open reduction and internal fixation (ORIF) as well as shoulder arthroplasty are the surgical treatment options for the management of dislocation of the shoulder with anatomical neck fracture [8,9]. One of the most feared complications (regardless of the fixation technique) is avascular necrosis (AVN) of the humeral head since it is a poorly vascularized area $[10,11]$. Schnetzke et al. [12] reported that late surgery (defined as surgery time $>48 \mathrm{~h}$ after sustained trauma) along with substandard/suboptimal reduction of the fracture are associated with a significant risk of AVN of the humeral head. Subsequently, there is an increased chance for revision surgeries. Although ORIF is associated with a greater risk of AVN, it allows preservation of the humeral head. The rationale behind choosing ORIF as a surgical option for the management of shoulder dislocation with concomitant anatomical neck fracture is that the symptoms of AVN of the humeral head will present late since the glenohumeral joint is a non-weight-bearing joint. Moreover, even if AVN of the humeral head develops, the overall function of the shoulder will not be compromised $[6,13,14]$. On the other hand, certain orthopedic surgeons do not prefer ORIF for the management of such injuries. They debate that the fixation of the humeral head in the context of anatomical neck fracture is difficult to attain and secure since it is a cartilage shell associated with a thin layer of bone. Furthermore, they claim that the prolonged immobilization for adequate union is associated with increased risk of the development of a stiff shoulder. They assert that hemiarthroplasty provides a more successful rehabilitation and that range of motion can be initiated earlier than usual $[1,5,15]$. Consequently, the literature remains controversial regarding the optimal surgical management. Moreover, it is advised that the location, size, and site of fracture fragments be taken into consideration during decision-making for surgical management [5]. Preoperative computed tomography (CT) with 3D reconstruction provides a better visualization of the fracture pattern which reflects positively in the decision-making process and in preoperative planning [5]. Herein, we present the case of a 33-year-old male patient presenting with anterior fracture dislocation of the shoulder with anatomical neck fracture.

\section{Case Report}

A 33-year-old male, right-hand-dominant, presented to the emergency department in 2016 after sustaining a traumatic event to his right shoulder following a snowboard accident. Upon presentation, the patient was conscious and denied any sustained trauma to the head. He was supporting his right elbow with his left hand and any attempted movement of his right shoulder elicited severe pain. Physical examination revealed small, superficial abrasions on 


\section{Case Reports in Orthopedic Research}

Case Rep Orthop Res 2020;3:108-117

DOI: 10.1159/000509818

c) 2020 The Author(s). Published by S. Karger AG, Basel www.karger.com/cio

Maalouly et al: Fracture Dislocation of the Anatomical Neck of the Proximal Humerus

his right hemithorax and right shoulder areas. However, there were no open wounds. Moreover, swelling and ecchymoses were noted over the lateral aspect of his right shoulder. The neurovascular examination of the patient revealed noncompromised vasculature as well as preserved motor strength and sensation in all four extremities. Radiographs of the right shoulder showed an empty glenoid and anterior dislocation of the humerus with a fracture of its anatomical neck showing marked overriding of the fragments as well as lateral displacement (Fig. 1). Subsequently, CT of the right shoulder in axial, sagittal, and coronal planes with 3D reconstruction (Fig. 2) was requested, showing a comminuted displaced fracture involving the right humeral neck associated with anterior dislocation of the right shoulder. The CT also revealed a small bony fragment adjacent to the glenoid posteriorly (Fig. 3, 4).

\section{Surgical Technique}

The injury to operating room time for this patient was $<8 \mathrm{~h}$. Under general anesthesia, the patient was put in the beach chair position, strapped, and draped. He was given $2 \mathrm{~g}$ of cefazolin within 30 min of incision as prophylactic antibiotics. The strategy was to relocate the humeral head with minimal dissection and in one piece if possible, with perfect anatomical reduction and stable fixation. Using the deltopectoral approach, the biceps was located and then a proximal tenotomy, closure to the labrum, from the glenoid was done. We identified the rotator interval and completely opened it without any damage to the subscapularis. Digital pressure over the anterior aspect of the humeral head was attempted to allow relocation, but without success. It was opted to avoid prolonged manipulation of the humeral due to the tenuous blood supply, so using the rotator cuff tear that was identified, a periosteal elevator (Cobb) was introduced into the glenohumeral joint. With gentle traction over the humerus distally and proximally with the periosteal elevator, reduction was achieved with the help of a bone holding forceps which was then temporarily fixed with two K-wires. Adequate reduction and alignment was seen on axillary and lateral views. The plate was placed slightly lateral and posterior to the bicipital groove, and one screw was placed in the elongated hole to fix it such as to allow fine-tuning of the final position, $6 \mathrm{~mm}$ distal to the greater tuberosity tip, aligned properly over the shaft using two fingers. Through appropriate drill sleeve, $3.5-\mathrm{mm}$ screws were inserted carefully to reach the subchondral bone without damaging the cartilage and after measuring appropriate length. The K-wires were removed. The screws were inserted in multiple directions and tenodesis of the biceps was done while as rotator cuff tear was identified and repaired using two anchors with restoration of anatomical coverage of the humeral head (Fig. 5). The operation time was approximately $1 \mathrm{~h}$ and $20 \mathrm{~min}$ with an estimated blood loss of $<250 \mathrm{~mL}$. The postoperative hospital course was uneventful and the drain was removed on postoperative day 2. The patient was discharged on day 3 to be followed up by his orthopedic surgeon as an outpatient.

\section{Follow-Up}

The patient was followed for 3 years post ORIF for his right proximal humerus shoulder fracture dislocation. The now 36-year-old male patient is fully recovered, with a normal range of motion of his right glenohumeral joint equal to the range of motion seen with his left shoulder joint (Fig. 6). The patient returned to his normal activity of daily living and job with no complaints or difficulties. In the correct clinical picture, the classic diagnostic sign for AVN of the humeral head is the crescent sign seen on radiographs [16]. However, the patient showed no signs of AVN of the humeral head throughout the course of the 3-year follow-up, as can be seen on X-ray (Fig. 7). 


\section{Case Reports in Orthopedic Research}

\section{Discussion}

Anatomical neck fractures of the humerus are infrequently reported injuries in the literature [17]. Henceforth, the literature does not contain clear, evidence-based guidelines for the surgical approach and operative management of such injuries. Since they are rare injuries, the diagnosis may be delayed or even missed. Moreover, dislocation of the glenohumeral joint with anatomical neck fracture is associated with a significantly high risk of AVN due to its poor vascularity. Hence, it is crucial for surgical intervention to be performed as early as possible. Therefore, early and correct diagnosis is important to prevent any delay in management. Neer [18] suggested that direct open reduction be favored over closed reduction to prevent further damage to the already compromised vascularity of the humeral head. The case presented in this study involved a 33-year-old male patient with an anteriorly dislocated glenohumeral joint associated with a laterally displaced fracture of the anatomical neck of the humerus. Time to surgery is of critical importance and early operative management of anatomical neck fracture of the humerus is associated with a significantly lower risk of AVN [12]. Delayed surgery leads to difficulty in the reduction of the fracture, increases the risk of AVN, and increases the risk of instability for secondary surgery [19]. In the presented case, the time from when the patient suffered from the trauma to when he was in the operation room was $<8 \mathrm{~h}$. Moreover, delay in diagnosis or misdiagnosis of anatomical neck fracture of the humerus can also be due to inadequate imaging [1]. In our case, X-rays of the right shoulder were obtained in the anteroposterior, lateral, and en-Y views as initial imaging modality. Preoperative CT is advised since it allows for better visualization of the fracture pattern, thus leading to a more accurate diagnosis. Moreover, CT helps in the decision-making process of choosing the adequate surgical approach. In the case of our patient, CT confirmed the X-ray result, showing a comminuted displaced fracture of the anatomical neck of the right humerus associated with an anteriorly dislocated shoulder. As for the surgical management, the patient underwent ORIF. ORIF is associated with an increased risk of AVN; however, this surgical approach allows preservation of the humeral head. The rationale behind choosing such an approach is that the symptoms of AVN present late and that the glenohumeral joint is not a weight-bearing joint, which would allow adequate recovery. The patient was followed for a period of 3 years, during which the patient showed full recovery and a normal range of motion of his right shoulder. He was followed with serial X-rays of his right shoulder which repetitively disclosed the absence of AVN. Moreover, the patient did not show signs or symptoms of AVN of the proximal humerus and he did not require any revision surgeries. Three years postoperatively, the patient has full range of motion of his right shoulder and shows complete uneventful recovery of the proximal humeral fracture. We believe that our success is due to the early time of surgical repair, the young age of the patient with good bone quality, minimal dissection and devascularization in our technique, and finally proper rigid fixation. As per the literature, it is advised for the orthopedic surgeon to properly assess each individualized case before attempting any surgical approach, since anatomical neck fractures are unrecognized in most cases of tuberosity fracture dislocation of the shoulder [17].

\section{Conclusion}

Fracture dislocation of the proximal humerus is a rare entity that is very challenging technically in young patients. It is vital to do the surgery as soon as possible after initial trauma for the best results. Intraoperatively careful manipulation of the humeral head is key for proper 
alignment and reduction with minimal disruption of vascularity while achieving rigid fixation. We present this case to show our management of such a difficult fracture and the ensuing excellent results on follow-up.

\section{Statement of Ethics}

Approval and consent of the ethics committee were received for the publication of this article. Full written well-informed approval for his case to be included in this study and for publication of images was given by the patient. No identifiers are included in this article relating to the patient's identity.

\section{Conflict of Interest Statement}

The authors have no conflicts of interest to disclose.

\section{Funding Sources}

The authors declare that there was no financial support or sponsorship for this study.

\section{Author Contributions}

J. Maalouly contributed to the writing and editing of this article. D.K. Aouad contributed to the writing of this article and to the submission process. A. Tawk contributed to the writing and referencing of this article. G. El Rassi contributed with the case as well as with the writing and editing of this article.

\section{References}

1 Ide J, Honda K, Takagi K. Posterior dislocation of the shoulder associated with fracture of the humeral anatomical neck with 11-year follow-up after early open reduction and internal fixation. Arch Orthop Trauma Surg. 2003 Apr;123(2-3):118-20.

2 Zacchilli MA, Owens BD. Epidemiology of shoulder dislocations presenting to emergency departments in the United States. J Bone Joint Surg Am. 2010 Mar;92(3):542-9.

3 Hohmann E, Tetsworth K, Glatt V. Open versus arthroscopic surgical treatment for anterior shoulder dislocation: a comparative systematic review and meta-analysis over the past 20 years. J Shoulder Elbow Surg. 2017 Oct;26(10):1873-80.

4 Hobby J, Griffin D, Dunbar M, Boileau P. Is arthroscopic surgery for stabilisation of chronic shoulder instability as effective as open surgery? A systematic review and meta-analysis of 62 studies including 3044 arthroscopic operations. J Bone Joint Surg Br. 2007 Sep;89(9):1188-96.

5 Tanwar YS, Jaiswal A, Kharbanda Y, Panda A. Posterior fracture dislocation of shoulder with anatomical neck fracture. Apollo Med. 2018 Apr;15(2):75-78.

6 Siavashi B, Golbakhsh MR. A case report of isolated posterior fracture dislocation of anatomical neck of humerus. Orthop Muscular Syst. 2012;1:116.

7 Kuner EH. Fractures of the proximal humerus. Classification and treatment principles. Z Unfallchirurgie Versicherungsmedizin. 1992;85(3):156-62.

8 Hessmann MH, Blum J, Hofmann A. Küchle R, Rommens PM. Internal fixation of proximal humeral fractures: current concepts. Eur J Trauma. 2003 Oct;29(5):253-61. 


\section{Case Reports in Orthopedic Research}

\begin{tabular}{l|l}
\hline DOI: 10.1159/000509818 & $\begin{array}{l}\text { (c) 2020 The Author(s). Published by S. Karger AG, Basel } \\
\text { www.karger.com/cio }\end{array}$ \\
\hline
\end{tabular}

Maalouly et al.: Fracture Dislocation of the Anatomical Neck of the Proximal Humerus

9 Kralinger F, Schwaiger R, Wambacher M, Farrell E, Menth-Chiari W, Lajtai G, et al. Outcome after primary hemiarthroplasty for fracture of the head of the humerus. A retrospective multicentre study of 167 patients. J Bone Joint Surg Br. 2004 Mar;86(2):217-9.

10 Greiner S, Kääb MJ, Haas NP, Bail HJ. Humeral head necrosis rate at mid-term follow-up after open reduction and angular stable plate fixation for proximal humeral fractures. Injury. 2009 Feb;40(2):186-91.

11 Hertel R, Hempfing A, Stiehler M, Leunig M. Predictors of humeral head ischemia after intracapsular fracture of the proximal humerus. J Shoulder Elbow Surg. 2004 Jul-Aug;13(4):427-33.

12 Schnetzke M, Bockmeyer J, Loew M, Studier-Fischer S, Grützner PA, Guehring T. Rate of avascular necrosis after fracture dislocations of the proximal humerus: timing of surgery. Obere Extrem. 2018;13(4):273-8.

13 Ogawa K, Yoshida A, Inokuchi W. Posterior shoulder dislocation associated with fracture of the humeral anatomic neck: treatment guidelines and long-term outcome. J Trauma. 1999 Feb;46(2):318-23.

14 Muller B, Bonaire F, Kuner EH. Dislocated fractures of the proximal humerus: treatment concepts, technique and results. Aktuelle Traumatol. 1998;28:261-70.

15 Bono CM, Renard R, Levine RG, Levy AS. Effect of displacement of fractures of the greater tuberosity on the mechanics of the shoulder. J Bone Joint Surg Br. 2001 Sep;83(7):1056-62.

16 Pappas JN. The musculoskeletal crescent sign. Radiology. 2000 Oct;217(1):213-4.

17 Mahmoud A, Diab R, Mostafa Abdeldayem S. Humeral neck fractures in association with traumatic shoulder dislocation. Orthop Rheumatol Open Access J. 2018;10(1):1-5.

18 Neer CS 2nd. Displaced proximal humeral fractures. II. Treatment of three-part and four-part displacement. J Bone Joint Surg Am. 1970 Sep;52(6):1090-103.

19 Godenèche A, Freychet B, Gunst S, Daggett M, Viste A, Walch G. Arthroscopic reduction of acute traumatic posterior glenohumeral dislocation and anatomic neck fracture without internal fixation: 2-year follow-up. Orthop J Sports Med. 2017 Dec;5(12):2325967117745486.

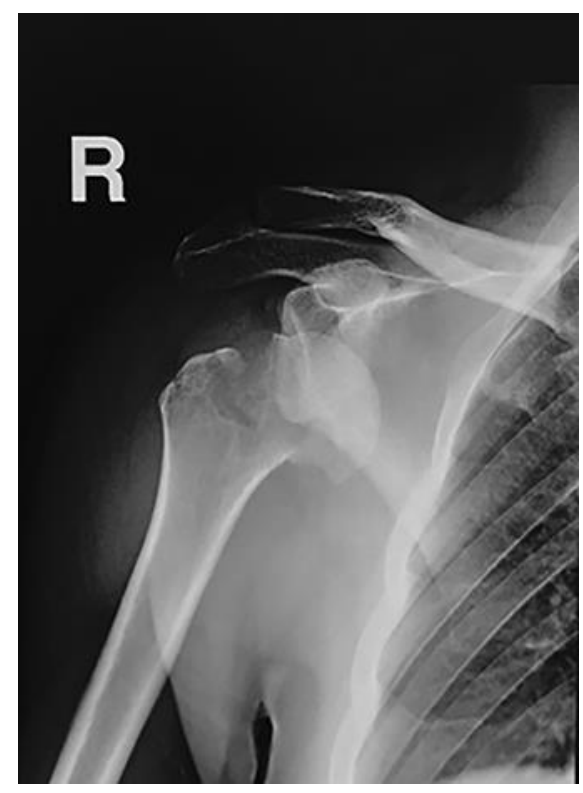

Fig. 1. Anteroposterior plain radiograph of the right shoulder showing an anteriorly dislocated shoulder associated with fracture of the anatomical neck of the humerus. 
Case Reports in Orthopedic Research
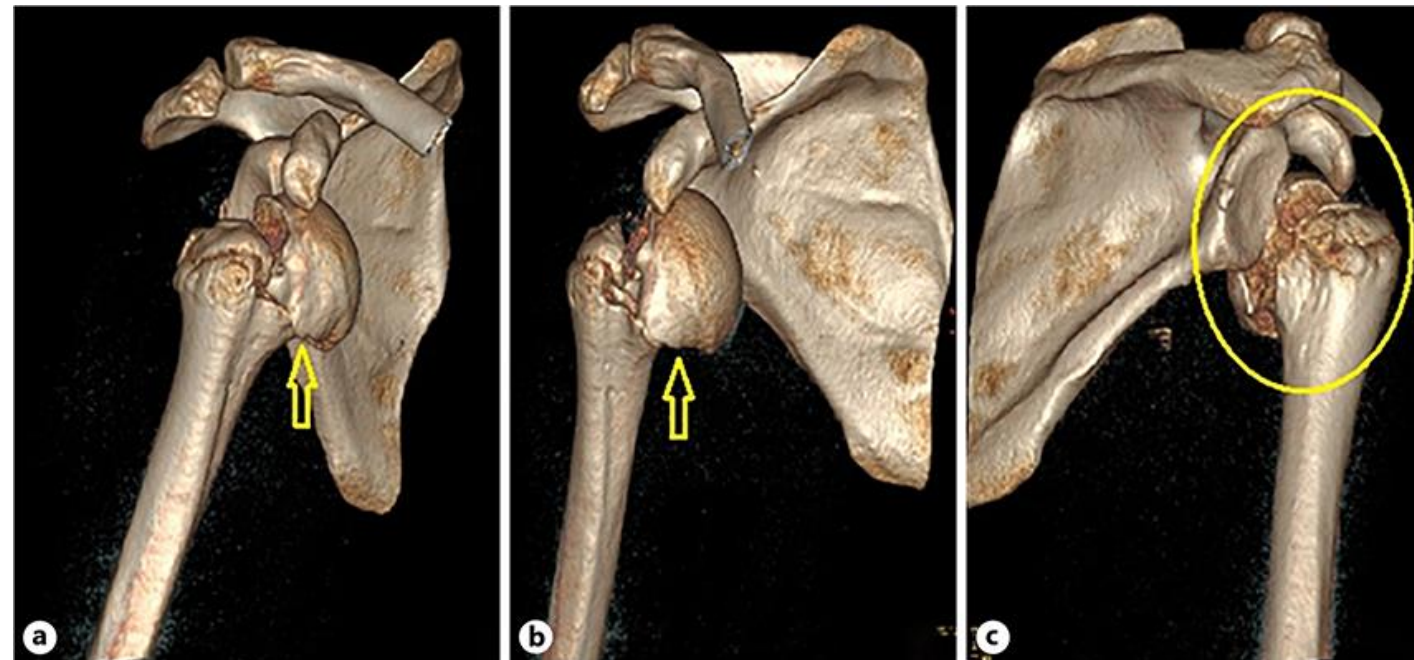

Fig. 2. a-c Computed tomography with 3D reconstruction of the right shoulder showing the anatomical neck fracture and dislocation.
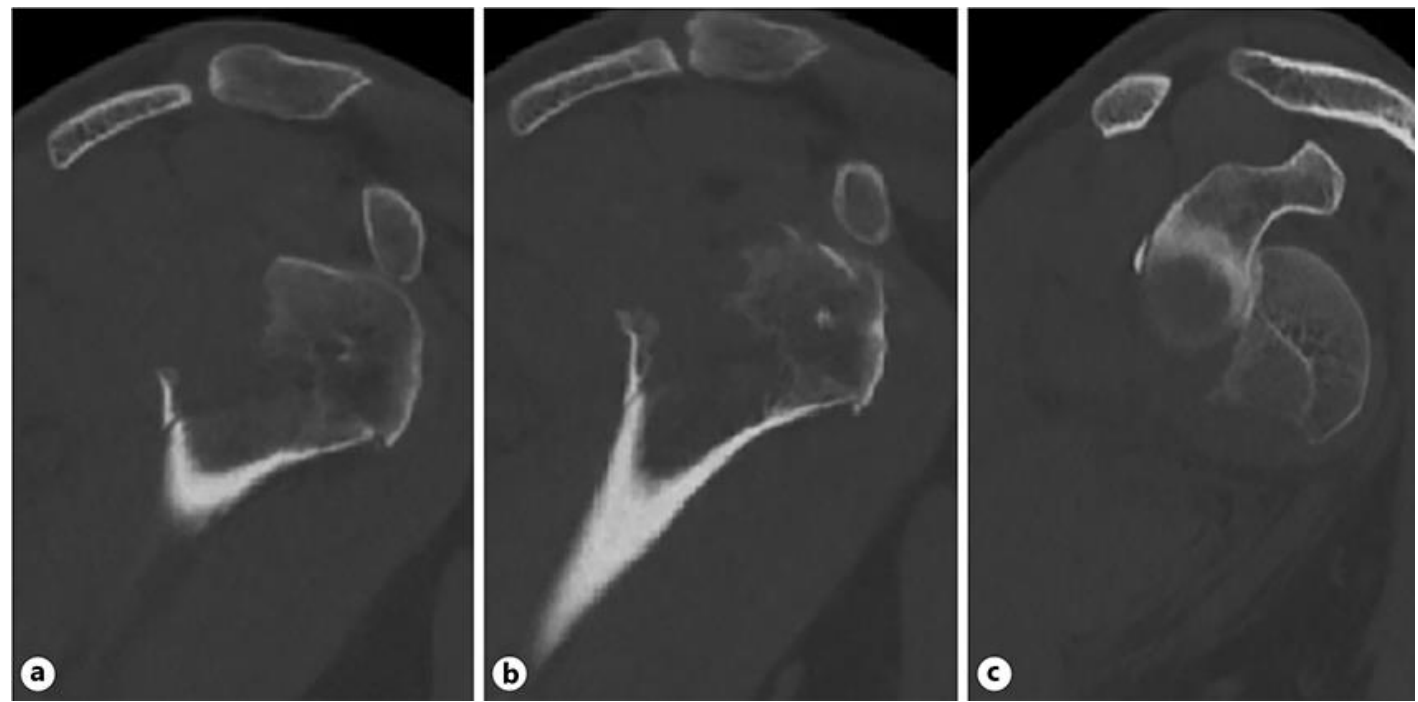

Fig. 3. a-c Computed tomography sagittal cuts of the right shoulder showing the anatomical neck fracture and dislocation. 
Case Reports in Orthopedic Research
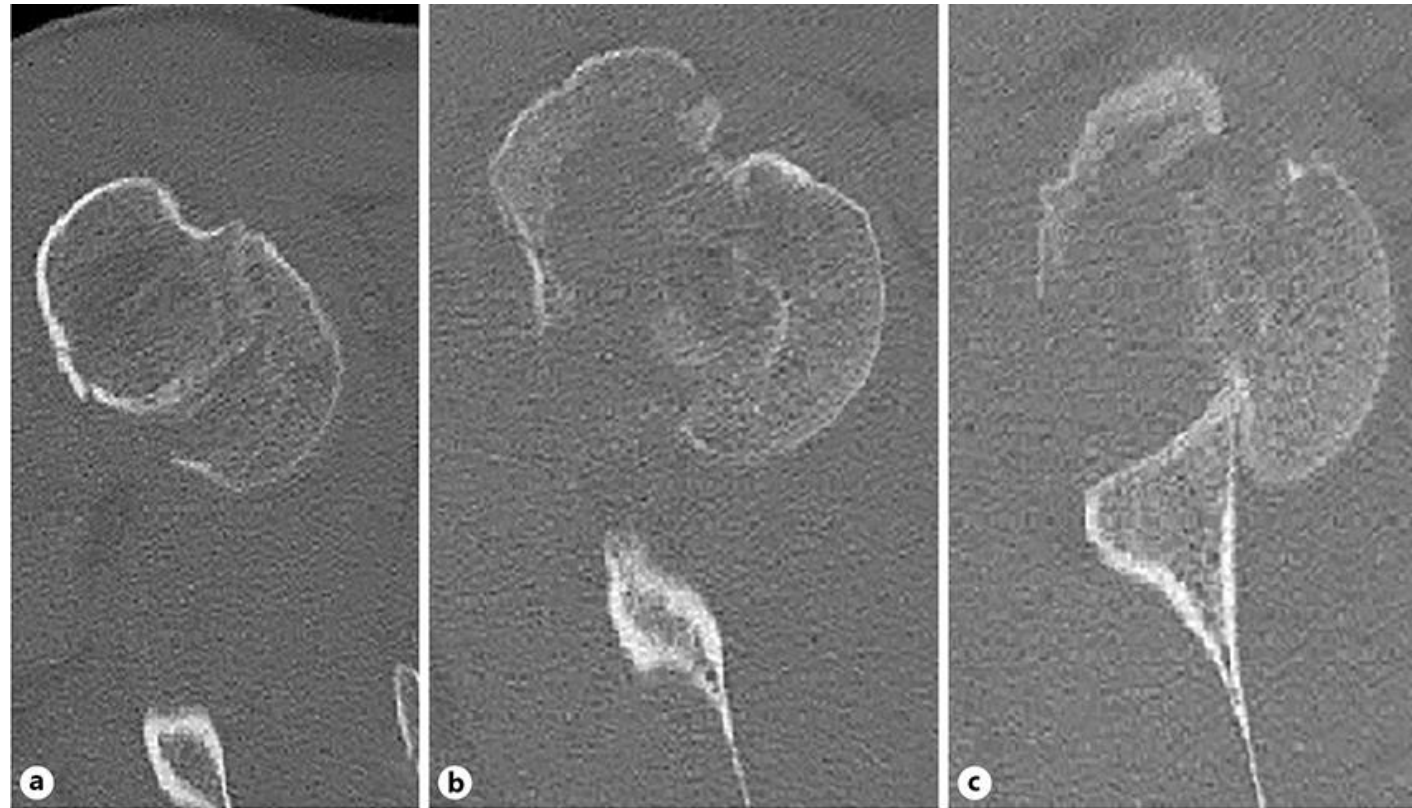

Fig. 4. a-c Computed tomography axial cuts of the right shoulder showing the anatomical neck fracture and dislocation.

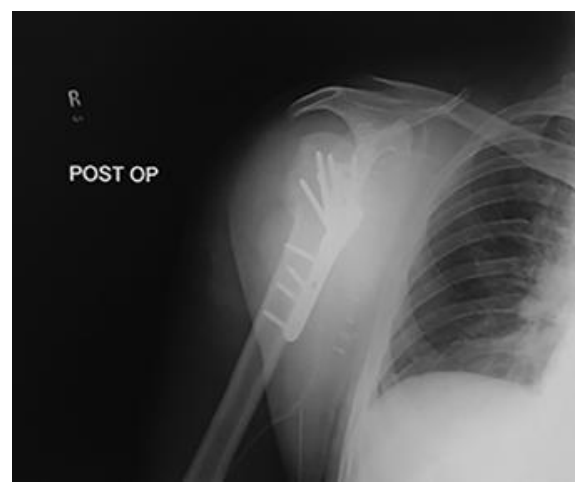

Fig. 5. Postoperative radiograph of the right shoulder showing satisfactory reduction after open reduction and internal fixation. 
Case Reports in Orthopedic Research
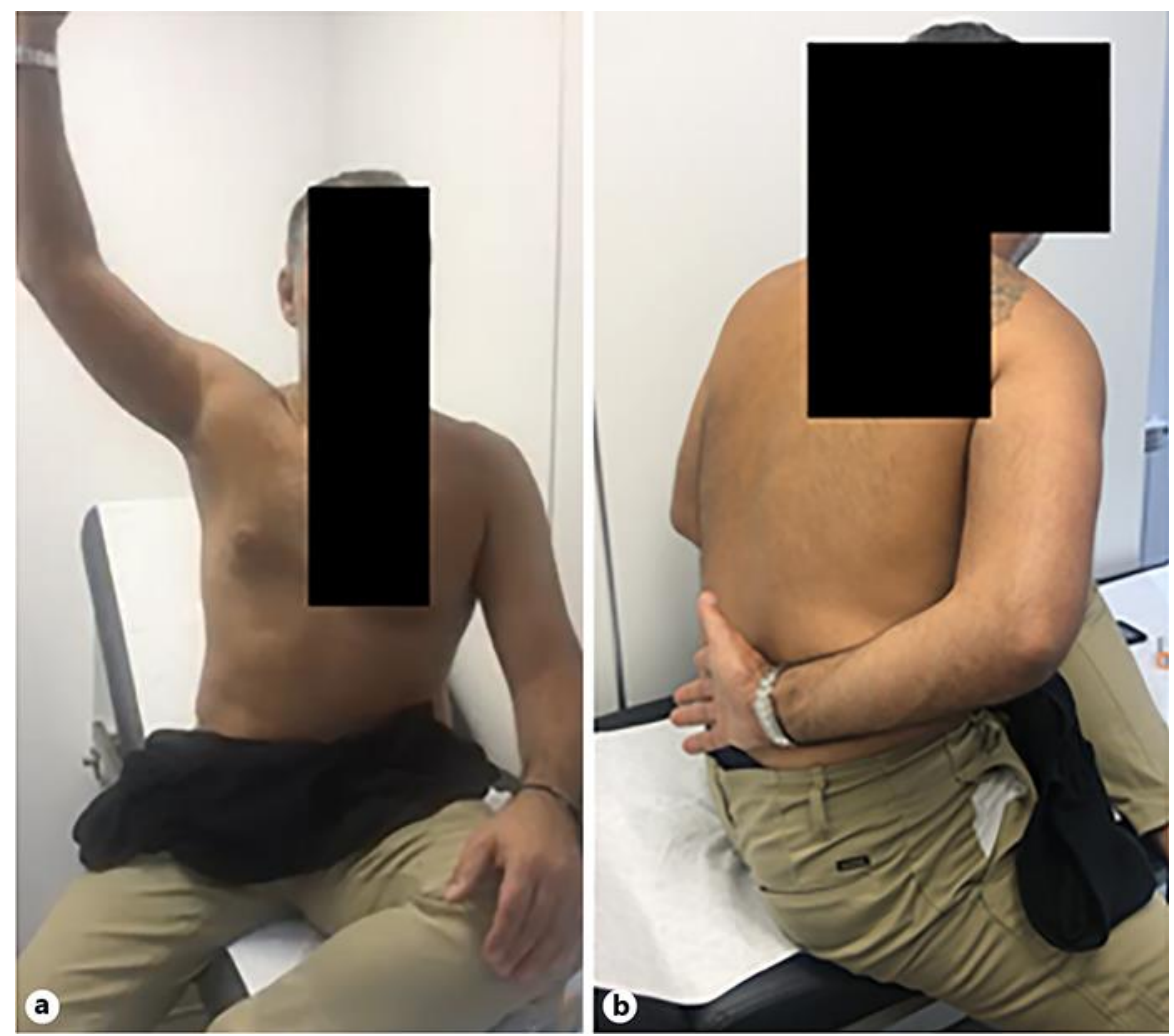

Fig. 6. Clinical examination at 3-year follow-up. a Lateral abduction $>90^{\circ}$ and external rotation. $\mathbf{b}$ Internal rotation. \begin{tabular}{l|l}
\hline DOI: $10.1159 / 000509818$ & ( 2020 The Author(s). Published by S. Karger AG, Basel \\
\hline
\end{tabular} www.karger.com/cio

Maalouly et al.: Fracture Dislocation of the Anatomical Neck of the Proximal Humerus (b) 
Case Reports in Orthopedic Research

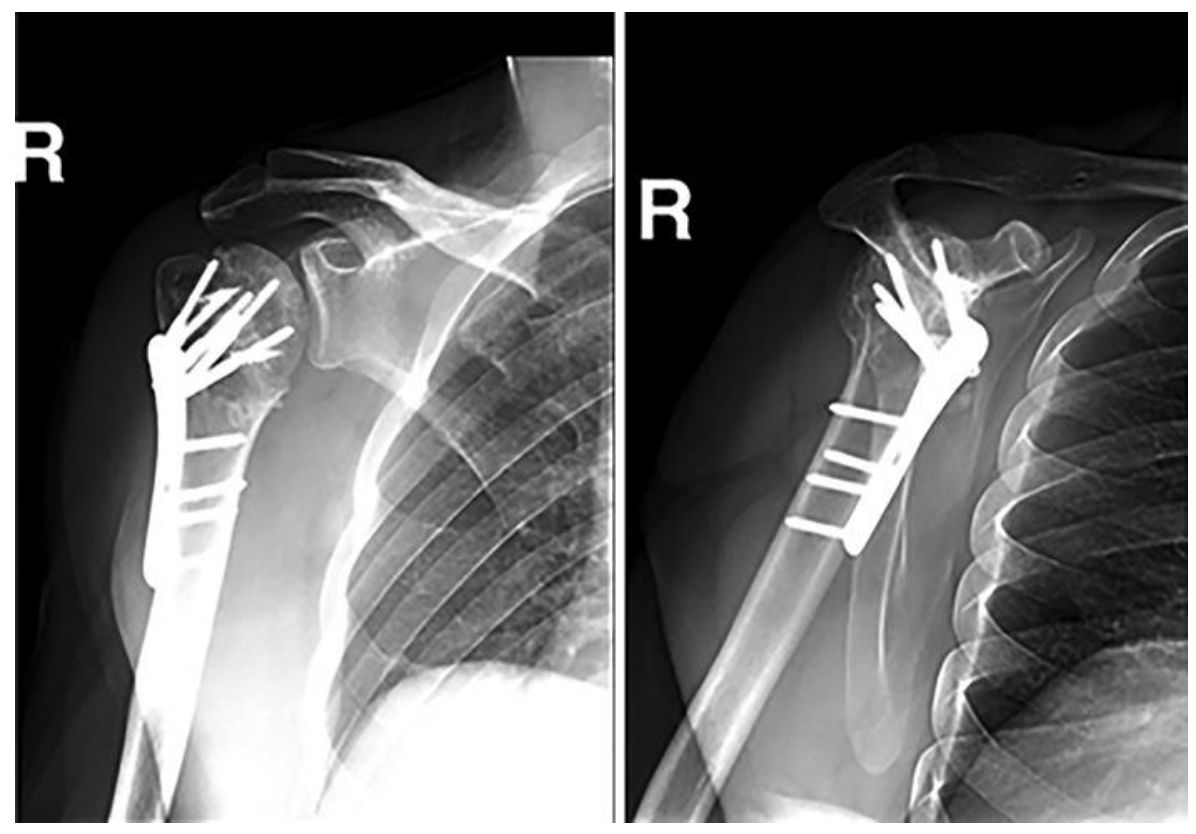

Fig. 7. X-ray of the right shoulder with multiple views on follow-up 3 years postoperatively showing satisfactory alignment and healed fracture. www.karger.com/cio

Maalouly et al.: Fracture Dislocation of the Anatomical Neck of the Proximal Humerus 